\title{
Induced displacive transition in heterogeneous materials
}

\author{
M. Apostol ${ }^{1,2, a}$, S. Ilie ${ }^{2,3}$, A. Petrut ${ }^{3}$, M. Savu ${ }^{2}$, and S. Toba ${ }^{3}$ \\ ${ }^{1}$ Department of Theoretical Physics, Institute of Atomic Physics, Magurele-Bucharest MG-6, PO Box MG-35, Romania \\ ${ }^{2}$ MIRA Technologies Ltd., Teiul Doamnei 2, Bucharest, Romania \\ ${ }^{3}$ MIRA Telecom, Grigorescu 13, 075100 Otopeni, Bucharest, Romania
}

Received: 8 May 2012 / Received in final form: 31 May 2012 / Accepted: 31 May 2012

Published online: 11 July 2012 - (C) The Author(s) 2012

\begin{abstract}
A model of heterogeneous, composite material is introduced, consisting of randomly distributed identical structural micro-domains endowed with electric charges or dipoles. Two cases are presented, one corresponding to a tightly packed (dense) material, another corresponding to highly-dispersed, small domains. The polarizability is computed in both cases, under the action of an external uniform electric field oscillating in time (a quasi-stationary field), and it is related to the displacement of the micro-domains from their positions of local equilibrium (translations or rotations). It is shown that the polarizability (or electric susceptibility) can exhibit characteristic (resonance) frequencies in the radio-frequency range and, even for moderate external fields, the material can undergo a displacive transition (similar to a ferroelectric transition), governed by non-linearities in the interaction energy of the micro-domains. The shift in the characteristic frequencies of the polarizability is estimated, as caused by the displacive modification.
\end{abstract}

\section{Introduction}

Most of the commonly used materials become heterogeneous, even if they are made of one chemical substance [1]. There are a large variety of heterogeneity types, referred to by a great deal of terms (composites, suspensions, colloids, polymers, blends, multi-phase alloys, etc.). Basically, in a simplified picture, such materials exhibit structural micro (or meso)-domains (grains, crystallites, etc.), which may appear naturally in the process of fabrication (grinding, milling, thermal treatments, etc.). The micro-domains, as well as their walls, may have their own dynamics (fluctuations included) [2]. One of the most interesting properties is the accumulation (segregation) of electric charge at the interfaces. Sometimes, this feature is known as the Maxwell-Wagner-Sillars phenomenon, and it is associated with an appreciable dielectric loss [3-7]. "In the heterogeneous materials there is usually an interfacial polarization arising from the accumulation of charge at structural interfaces. This is of little fundamental interest, but it is of considerable practical interest because commercial insulating materials are usually heterogeneous" $[8,9]$. We show here that the electric polarization of the domains in heterogeneous materials can exhibit interesting properties at the fundamental level, with surprising practical implications. Specifically, we show that a displacive transition of the domain polarization can occur in such materials, induced by an external field, leading to a remanent polar-

\footnotetext{
${ }^{a}$ e-mail: apoma@theory.nipne.ro
}

ization (and a hysteresis loop), whose dynamics is investigated here.

The dielectric properties of such composite materials have ever enjoyed a great deal of interest. On one hand, these materials have a wide range of applications, such as shielding enclosures, electromagnetic absorbants, antistatic devices, capacitive memory disks, etc. [10]. On the other hand, there are many theoretical investigations devoted to the effective electric susceptibility of such materials [11-16]. Such studies aim, generally, at relating the macroscopic electromagnetic properties to the internal micro-structure, and, especially, understanding the role played by interfaces in the dielectric properties. Ferromagnetic alignment has recently been demonstrated for nano-inclusions embedded in bulk superconductors [17]. Fractal structure of composite materials, especially structured interfaces, has been exploited for non-linear electric conductivity of Josephson junctions, with potential applications in radio-physical devices [18]. Composite materials with radioactive inclusions have been designed, amenable to controlling the electromagnetic properties, particularly the dielectric function [19]. A great deal of work is done for relating the dielectric function of a composite to the dielectric functions of its components, as well as for characterizing various relaxation mechanisms and their implications. We introduce here a model of randomly distributed micro-domains, either densely packed or highly dispersed, endowed with electric charges or dipoles, and investigate the dynamics of these micro-domains under 
the action of an external uniform electric field oscillating in time. We show, on one side, that the electric polarizability (or susceptibility) can exhibit characteristic (resonance) frequencies in the radio-frequency range and, on the other, that these micro-domains can suffer a displacive transition under the action of the external field (translations for charges, rotations for dipoles), with a remanent polarization (and a hysteresis loop), very much alike a ferroelectric material.

\section{Polarization and electric susceptibility}

We assume a simple model of identical micro-domains of (linear) size $d$, mass $M$ and charge $\pm Q$, separated by a mean distance $D(D>d)$ and randomly distributed in an infinite (composite) solid, in a metastable equilibrium. By analogy with an ionic solid, we assume that the potential energy of each domain can be written as $U=C u^{2} / 2$, where $u$ is the small displacement of the the domain from its (local) equilibrium position and the force constant $C$ is of the order of $C=Q^{2} / D^{3}$. It is easy to see that such an interaction energy introduces a characteristic oscillation frequency $\Omega_{0}$ given by:

$$
\Omega_{0}^{2}=\frac{Q^{2}}{M D^{3}}
$$

The charge $Q$ is usually accumulated on the surface of the micro-domains, on a number $N_{s}$ of atoms, of the order of the number of atoms on the surface. It is convenient to refer this charge to the electron charge $e$, and write $Q=e N_{s}$. Similarly, it is convenient to refer the microdomain mass $M$ to a mass $M_{0}$ of the order of the atomic mass, and write $M=M_{0} N$, where $N$ is the number of atoms in the micro-domain. Since $d^{3}$ is of the order of the domain volume, we may write also $d^{3}=a^{3} N$, where $a$ is of the order of the atomic interparticle separation distance. From equation (1) we get:

$$
\Omega_{0}=\omega_{0}\left(\frac{d}{D}\right)^{3 / 2} \frac{N_{s}}{N} \simeq \omega_{0}\left(\frac{d}{D}\right)^{3 / 2} \frac{a}{d},
$$

where $\omega_{0}=\sqrt{e^{2} / M_{0} a^{3}}$ can be viewed as an (atomic) plasma frequency. Typically, $\omega_{0}$ is of the order of $1-10 \mathrm{THz}$. We can see that the factors intervening in equation (2) can reduce this frequency considerably. We distinguish two cases: $d \simeq D$ and $d \ll D$. The former case corresponds to a densely packed composite, with quasi-adjoining microdomains (high filling factor). The latter case corresponds to highly dispersed micro-domains, of small size, separated by an electrically quasi-inert medium (low filling factor). In the first case we may take $d \simeq D=100 \mu \mathrm{m}$ $\left(N_{s} / N \simeq 10^{-6}\right)$ and get a characteristic frequency $\Omega_{0} \simeq$ $10^{6}-10^{7} \mathrm{~Hz}$, i.e., in the radio-frequency range. For $d \ll D$ we may take $d \simeq a$ and $D=1 \mu$ for instance, and the resulting characteristic frequency is again $\Omega_{0} \simeq 10^{6}-10^{7} \mathrm{~Hz}$ in the radio-frequency range. Equation (2) implies a large variability in the characteristic frequency $\Omega_{0}$, depending on the parameters $d$ and $D$, with the general feature that this characteristic frequency is lowered to a large extent in comparison with the the atomic-scale frequencies $\omega_{0}$.

The equation of motion for a micro-domain (of charge $Q$ ) under the action of an external, uniform electric field $E_{0}$ can be written as:

$$
M \ddot{u}=Q E_{t}-M \Omega_{0}^{2} u-M \gamma \dot{u},
$$

where $\gamma$ is a damping parameter and $E_{t}=E_{0}+E$ is the total electric field, $E$ being the internal (polarization) electric field. Equation (3) refers to the relative displacement of a micro-domain with respect to its surroundings, so that $M$ should be viewed as the reduced mass of two (oppositely charged) micro-domains; it is of the same order of magnitude as the mass of a single microdomain. By Fourier transform, we get from equation (3) the displacement:

$$
u(\omega)=-\frac{Q E_{t}(\omega)}{M} \times \frac{1}{\omega^{2}-\Omega_{0}^{2}+\mathrm{i} \omega \gamma} .
$$

According to the classical electromagnetism, for $d \simeq D$ the polarization is given by:

$$
P(\omega)=\frac{Q u(\omega)}{D^{3}}=-\frac{\Omega_{p 1}^{2}}{4 \pi} \times \frac{E_{t}(\omega)}{\omega^{2}-\Omega_{0}^{2}+i \omega \gamma},
$$

where

$$
\Omega_{p 1}=\sqrt{\frac{4 \pi Q^{2}}{M D^{3}}},
$$

is the well-known plasma frequency (for charged microdomains) and the factor $4 \pi$ has been introduced according to the usual conventions. We can see that $\Omega_{p 1}$ is of the same order of magnitude as the characteristic frequency $\Omega_{0}$ given by equation (1).

For small micro-domains $(d \ll D)$ highly dispersed in an electrically quasi-inert medium $d \ll D$, the polarization is given by:

$$
P(\omega)=\frac{Q u(\omega)}{d^{3}}=-\frac{\Omega_{p 2}^{2}}{4 \pi} \times \frac{E_{t}(\omega)}{\omega^{2}-\Omega_{0}^{2}+i \omega \gamma},
$$

where

$$
\Omega_{p 2}=\sqrt{\frac{4 \pi Q^{2}}{M d^{3}}}=\Omega_{0}\left(\frac{D}{d}\right)^{3 / 2} .
$$

We can see that $\Omega_{p 2}$ can be much higher in this case than the characteristic frequency $\Omega_{0}\left(\Omega_{p 2} \gg \Omega_{0}\right.$; it may reach the $\mathrm{THz}$ range). Since the active, polarized micro-domains are low in number and have a small size, the internal field generated by them is vanishing, so we may take:

$$
P(\omega)=-\frac{\Omega_{p 2}^{2}}{4 \pi} \times \frac{E_{0}(\omega)}{\omega^{2}-\Omega_{0}^{2}+\mathrm{i} \omega \gamma},
$$

an equation which defines the polarizability (per unit volume)

$$
\alpha(\omega)=P(\omega) / E_{0}(\omega)=-\frac{\Omega_{p 2}^{2}}{4 \pi} \times \frac{1}{\omega^{2}-\Omega_{0}^{2}+i \omega \gamma} .
$$


It coincides with the electric susceptibility, which, in both cases, is given by:

$$
\chi(\omega)=P(\omega) / E_{t}(\omega)=-\frac{\Omega_{p 1,2}^{2}}{4 \pi} \times \frac{1}{\omega^{2}-\Omega_{0}^{2}+i \omega \gamma}
$$

(the polarizability for the first case $(d \simeq D)$ is computed in the next section).

It is worth noting that we discuss here two limiting cases $(d \simeq D$ and $d \ll D)$ for the micro-structure of a model composite, in order to bring to light salient features of the polarization dynamics associated with structural inhomogenities. In general, the polarization of the background medium containing the interspersed microdomains must be considered, for various filling factors, which implies more elaborate models and a more complex dynamics (including the loss mechanisms).

\section{Polarizability for a dense material}

As it is well known, with usual notations, the Maxwell equations read:

$$
\begin{aligned}
\operatorname{div} \mathbf{E}=-4 \pi \operatorname{div} \mathbf{P}, \quad \operatorname{div} \mathbf{H}=0, \\
\operatorname{curl} \mathbf{E}=-\frac{1}{c} \frac{\partial \mathbf{H}}{\partial t}, \quad \operatorname{curl} \mathbf{H}=\frac{1}{c} \frac{\partial \mathbf{E}}{\partial t}+\frac{4 \pi}{c} \frac{\partial \mathbf{P}}{\partial t},
\end{aligned}
$$

where the polarization charges $\left(\rho_{p}=-\operatorname{div} \mathbf{P}\right)$ and currents $\left(\mathbf{j}_{p}=\partial \mathbf{P} / \partial t\right)$ are explicitly introduced as sources for the internal fields $\mathbf{E}$ and $\mathbf{H}$. The polarization $\mathbf{P}$ in these equations, as well as the fields, are spatially averages, which are supposed to be meaningful for the case $d \simeq D$.

We take the curl of Faraday's equation in equation (12) and use curl $\times \operatorname{curl}=-\Delta+\operatorname{grad} \times$ div. Making use of Gauss's law for the electric field in equation (12) we get easily:

$$
\frac{1}{c^{2}} \frac{\partial^{2} \mathbf{E}}{\partial t^{2}}-\Delta \mathbf{E}=4 \pi \operatorname{grad} \times \operatorname{div} \mathbf{P}-\frac{4 \pi}{c^{2}} \frac{\partial^{2} \mathbf{P}}{\partial t^{2}},
$$

which is the wave equation (for the internal electric field) with polarization sources. We can expand the electric field and the polarization in equation (13) in series of eigenfunctions of the Laplacian (corresponding to the shape and the boundary conditions of the polarized piece of matter; in general, we leave aside the depolarizing fields associated with finite-size samples). For an infinite solid, we take the spatial Fourier transform of equation (13) (with respect to wavevectors $\mathbf{k}$ ), together with the temporal Fourier transform. Omitting for simplicity the arguments $\omega, \mathbf{k}$ this equation becomes:

$$
\left(\omega^{2}-c^{2} k^{2}\right) \mathbf{E}=4 \pi c^{2} \mathbf{k}(\mathbf{k P})-4 \pi \omega^{2} \mathbf{P} .
$$

We use this equation in conjunction with a more general definition of the electric susceptibility:

$\mathbf{P}(\omega, \mathbf{k})=\chi(\omega, \mathbf{k}) \mathbf{E}_{t}(\omega, \mathbf{k})=\chi(\omega, \mathbf{k})\left[\mathbf{E}_{0}(\omega, \mathbf{k})+\mathbf{E}(\omega, \mathbf{k})\right]$ which includes the spatial dispersion, i.e., the k-dependence. The algebraic equations (14) and (15) can be solved straightforwardly. Leaving again aside the arguments $\omega, \mathbf{k}$ for the moment we get the electric field:

$$
\mathbf{E}=-\frac{4 \pi \chi}{\varepsilon} \frac{\varepsilon \omega^{2} \mathbf{E}_{0}-c^{2} \mathbf{k}\left(\mathbf{k} \mathbf{E}_{0}\right)}{\varepsilon \omega^{2}-c^{2} k^{2}},
$$

and the polarization:

$$
\mathbf{P}=\frac{\chi}{\varepsilon \omega^{2}-c^{2} k^{2}}\left[\left(\omega^{2}-c^{2} k^{2}\right) \mathbf{E}_{0}+\frac{4 \pi \chi}{\varepsilon} c^{2} \mathbf{k}\left(\mathbf{k} \mathbf{E}_{0}\right)\right],
$$

where $\varepsilon=1+4 \pi \chi$ is the dielectric function. We can see from these equations the well-known polaritonic dispersion relation $\varepsilon \omega^{2}=c^{2} k^{2}$ for the propagation of the electromagnetic field in matter. We can also check the well-known relationship $\mathbf{D}=(\varepsilon / \chi) \mathbf{P}=\varepsilon \mathbf{E}_{t}$, where $\mathbf{D}=\mathbf{E}_{t}+4 \pi \mathbf{P}$ is the electric displacement, as well as the equality of the longitudinal components $\mathbf{k D}=\mathbf{k E}_{0}(\mathbf{k E}=-4 \pi \mathbf{k} \mathbf{P})$ of the electric displacement $\mathbf{D}$ and the external field $\mathbf{E}_{0}$.

For a uniform polarization and a uniform external electric field we take the limit $\mathbf{k} \rightarrow 0$ in the above equations. We get immediately:

$$
P(\omega)=\frac{\chi(\omega)}{\varepsilon(\omega)} E_{0}(\omega)=\frac{\chi(\omega)}{1+4 \pi \chi(\omega)} E_{0}(\omega),
$$

where $P(\omega)$ and $\chi(\omega)$ are given by equations (5) and (11), respectively. Equation (18) defines the polarizability:

$$
\begin{aligned}
\alpha(\omega) & =P(\omega) / E_{0}(\omega)=\frac{\chi(\omega)}{1+4 \pi \chi(\omega)} \\
& =-\frac{\Omega_{p 1}^{2}}{4 \pi} \times \frac{1}{\omega^{2}-\Omega_{0}^{2}-\Omega_{p 1}^{2}+\mathrm{i} \omega \gamma},
\end{aligned}
$$

we can see that it differs from the susceptibility by the renormalization of the characteristic frequency $\Omega_{0}^{2} \rightarrow \Omega_{0}^{2}+$ $\Omega_{p 1}^{2}$. We introduce the characteristic frequency $\Omega_{c}=$ $\sqrt{\Omega_{0}^{2}+\Omega_{p 1}^{2}}$, which is of the same order of magnitude as the frequencies $\Omega_{0, p 1}$ and write the polarizability as:

$$
\alpha(\omega)=-\frac{\Omega_{p 1}^{2}}{4 \pi} \times \frac{1}{\omega^{2}-\Omega_{c}^{2}+\mathrm{i} \omega \gamma} .
$$

Taking into account the results of the previous section, we use the general equation:

$$
\alpha(\omega)=-\frac{\Omega_{p}^{2}}{4 \pi} \times \frac{1}{\omega^{2}-\Omega_{c}^{2}+i \omega \gamma},
$$

where $\Omega_{p, c}$ are viewed as free parameters. For our present purpose we distinguish two cases, $\Omega_{p} \simeq \Omega_{c}(d \simeq D)$ and $\Omega_{p} \gg \Omega_{c}(d \ll D)$. The characteristic frequency $\Omega_{c}$ is in the radio-frequency range, while the plasma frequency $\Omega_{p}$ may get as high as the $\mathrm{THz}$ range (for $d \ll D$ ). Making use of equations (5) and (7), we can see that the displacement $u=D^{3} P / Q$ or $u=d^{3} P / Q$ is given by:

$$
u(\omega)=-\frac{Q E_{0}(\omega)}{M} \times \frac{1}{\omega^{2}-\Omega_{c}^{2}+\mathrm{i} \omega \gamma},
$$


in both cases. Equation (22) corresponds to the equation of motion of the form:

$$
\ddot{u}=\frac{Q E_{0}}{M}-\Omega_{c}^{2} u-\gamma \dot{u},
$$

which is similar to the equation of motion (3), except for the occurrence of the external field $E_{0}$ in place of the total field $E_{t}$ and the renormalization of the characteristic frequency $\Omega_{c}$ (for $\left.d \simeq D\right)$.

\section{Induced displacive transition}

It is worth estimating the amplitude of the displacement $u(t)$ under the action of an external field with a main Fourier component $\omega=\Omega_{c}$. Making use of equation (22) we get easily:

$$
u \simeq \frac{Q E_{0}\left(\Omega_{c}\right)}{M \Omega_{c}} \simeq \frac{Q E_{0}}{M \Omega_{c}} \Delta t
$$

for the amplitude of this displacement, where $E_{0}$ is the amplitude of the external field and $\Delta t$ is the duration of the wave packet centered on the frequency $\Omega_{c}$. For a moderate field $E_{0}=10^{3} \mathrm{~V} / \mathrm{m}\left(1 \mathrm{~V} / \mathrm{m}=(1 / 3) \times 10^{-4}\right.$ statvolt $\left./ \mathrm{cm}\right)$ and $\Omega_{c}=1 \mathrm{MHz} M_{0}=10^{4} \mathrm{~m}$ (where $m$ is the electron mass) we get $u(\mathrm{~cm}) \simeq 10^{6}\left(N_{s} / N\right) \Delta t(\mathrm{~s})$, which is a very large displacement. It is due mainly to the low value of the characteristic frequency $\Omega_{c}$ in comparison with "atomic" frequencies.

Under these circumstances the harmonic approximation in the equation of motion (3) is not valid anymore. The potential energy $M \Omega_{c}^{2} u^{2} / 2$ acquires higher-order nonlinear terms. It can be written as:

$$
U=\frac{1}{2} M \Omega_{c}^{2} u_{0}^{2}\left(v^{2}-A v^{4}+\frac{1}{3} B v^{6}\right),
$$

where $v=u / u_{0}, u_{0}$ is a scale length and $A, B>0$ are parameters. This potential energy has two minima, one for $v=0(U=0)$ and another for $v_{0}$ given by:

$$
v_{0}^{2}=\frac{1}{B}\left(A+\sqrt{A^{2}-B}\right),
$$

for $A^{2}>B$. This minimum energy can be either positive or negative, depending on the parameters $A$ and $B$. In both cases the material acquires a static displacement $u_{0} v_{0}$, and a corresponding static polarization $(P=$ $Q u_{0} v_{0} / D^{3}$ or $\left.P=Q u_{0} v_{0} / d^{3}\right)$, under the action of a threshold external field (or by other external means). The transition may either require energy or release energy. The material exhibits a displacive transition, similar to a ferroeletric transition. It is easy to see that the equation of motion for the coordinate $u$ (reduced coordinate $v$ ) written with an external field and the force derived from the potential $U$ given by equation (25) exhibits a characteristic bi-stable solution, which represents the well-known Landau-Devonshire hysteresis loop [20-25].
In the vicinity of $v_{0}$ the potential energy can be expanded in powers of the displacement $\xi$ around its minimum value; the quadratic term reads:

$$
U_{2}=\frac{1}{2} M \Omega_{c}^{2}\left(1-6 A v_{0}^{2}+5 B v_{0}^{4}\right) \xi^{2},
$$

which indicates a shift of frequency:

$$
\begin{aligned}
\Omega_{c} \rightarrow \widetilde{\Omega}_{c} & =\Omega_{c}\left(1-6 A v_{0}^{2}+5 B v_{0}^{4}\right)^{1 / 2} \\
& =2 \Omega_{c}\left[-1+\frac{A^{2}}{B}+\sqrt{\frac{A^{2}}{B}\left(\frac{A^{2}}{B}-1\right)}\right]^{1 / 2}
\end{aligned}
$$

It is reasonable to assume $A^{2} \geq B \gg 1$ (in order to have a small value for $v_{0}$ ). The new frequency is given in this case by:

$$
\widetilde{\Omega}_{c} \simeq 2 \Omega_{c}\left(A^{2} / B-1\right)^{1 / 4} .
$$

For $A^{2}=4 B / 3$, the potential energy does not change during the transition $\left(U_{0}=0\right)$ and the frequency remains unchanged $\left(\widetilde{\Omega}_{c}=\Omega_{c}\right)$.

For $A^{2} \geq B \gg 1$ the remanent polarization (zero field) corresponds to $v_{r} \simeq \sqrt{A / B}$ (close to the saturation polarization, which is obtained from the extreme of the total force) and the coercive field corresponds to $v_{c} \simeq$ $\sqrt{5}\left(A^{2} / B-1\right)$.

\section{A random distribution of dipoles}

Let us consider a random (or quasi-random) distribution of identical dipoles $\mathbf{p}$, of charge $Q$ and length $d$, separated by distances of the order of the distance $D(d \ll D)$. The total polarization can be zero or non-zero. Each dipole $\mathbf{p}_{1}$ is surrounded by identical dipoles, randomly oriented and randomly distributed around it, at distance $D$. It is reasonable to assume that the average over such a surrounding is a dipole $\mathbf{p}_{2}$, separated by the former dipole by the position vector $\mathbf{R}$ (whose magnitude is of the order of $D$ ). As it is well known, the interaction energy of the two dipoles is given by:

$$
U=\frac{1}{R^{5}}\left[\left(\mathbf{p}_{1} \mathbf{p}_{2}\right) R^{2}-3\left(\mathbf{p}_{1} \mathbf{R}\right)\left(\mathbf{p}_{2} \mathbf{R}\right)\right] .
$$

We introduce the angles $\alpha$ and $\beta$ between $\mathbf{R}$ and $\mathbf{p}_{1}$, respectively, $\mathbf{p}_{2}$, and the above energy becomes:

$$
\begin{aligned}
U & =\frac{p_{1} p_{2}}{R^{3}}[\cos (\alpha-\beta)-3 \cos \alpha \cos \beta] \\
& =\frac{p_{1} p_{2}}{D^{3}}(-2 \cos \alpha \cos \beta+\sin \alpha \sin \beta) .
\end{aligned}
$$

Let we assume that $\mathbf{p}_{2}$ is fixed, and $\mathbf{p}_{1}$ is in local equilibrium. Then, the derivative of $U$ with respect to $\alpha$ must vanish, $2 \sin \alpha \cos \beta+\cos \alpha \sin \beta=0$. Making use of this relation, the quadratic variation of $U$ becomes:

$$
U_{2}=\frac{p_{1} p_{2}}{D^{3}} \frac{(\delta \alpha)^{2}}{\sqrt{1+3 \sin ^{2} \alpha}} .
$$


The angle $\alpha$ is randomly distributed, so we can take the average with respect to this angle in equation (32) (which is of the order of unity); similarly, we can take approximately the same value $p$ for the magnitude of the two dipoles and get an interaction energy:

$$
U_{2} \simeq \frac{p^{2}}{D^{3}}(\delta \alpha)^{2}
$$

A change $\delta \alpha$ gives a force $-\left(2 p^{2} / d D^{3}\right) \delta \alpha$ and a torque $-\left(2 p^{2} / D^{3}\right) \delta \alpha$. Let us apply an external, uniform electric field $\mathbf{E}_{0}$, which makes an angle $\gamma$ with the dipole $\mathbf{p}$ (randomly oriented). The potential energy is $-p E_{0} \cos \gamma$. It gives a force $\left(p E_{0} / d\right) \sin \gamma$ and a torque $p E_{0} \sin ^{2} \gamma$. Its mean value is $\left(p E_{0} / 2\right)$. The equation of motion for the rigid body is then:

$$
I \ddot{\delta \alpha}=\frac{1}{2} p E_{0}-\frac{2 p^{2}}{D^{3}} \delta \alpha,
$$

where $I=M d^{2}$ is the momentum of inertia of the dipole (we neglect the relaxation). The angle variation $\delta \alpha$ implies a displacement $u=d \delta \alpha$, so that we get the equation of motion:

$$
\ddot{u}=\frac{p d E_{0}}{2 I}-\frac{2 p^{2}}{I D^{3}} u,
$$

and the displacement:

$$
u(\omega)=-\frac{p d E_{0}(\omega)}{2 I} \times \frac{1}{\omega^{2}-\Omega_{c}^{2}} .
$$

We can see that the characteristic frequency $\Omega_{c}=\sqrt{2 p^{2} / I D^{3}}=\sqrt{2 Q^{2} / M D^{3}}$ is of the same order of magnitude as the frequency $\Omega_{0}$ given by equation (1), or the characteristic frequency $\Omega_{c}=\sqrt{\Omega_{0}^{2}+\Omega_{p 1}^{2}}$, where $\Omega_{p 1}$ is defined by equation (6). It may lie very well in the radio-frequency range. It is worth noting the close analogy between the frequency $\Omega_{c}$ derived here and the (classical) Rabi frequency, if we put the electric field $E \simeq p / D^{3}$. (In quantum regime $I=L / \omega$ and the quantum of angular momentum $L$ is Planck's quantum $\hbar$; the frequency $\omega \simeq \sqrt{p E / I}$ given above becomes $\omega=p E / \hbar$, i.e., the frequency of a spatial rotator, as it is well known [26].)

The projection of a dipole $\mathbf{p}$ along the direction of the external field $\mathbf{E}_{0}$ is $p \cos \gamma$. Its variation gives a dipole momentum $p|\sin \gamma| \delta \alpha$ which signifies a mean polarization:

$$
P \simeq \frac{p}{d^{4}} u=-\frac{p^{2} E_{0}(\omega)}{2 I d^{3}} \times \frac{1}{\omega^{2}-\Omega_{c}^{2}},
$$

which defines a "plasma" frequency given by:

$$
\Omega_{p}^{2}=\frac{2 \pi p^{2}}{I d^{3}}=\frac{2 \pi Q^{2}}{M d^{3}} .
$$

It is of the same order of magnitude as the plasma frequency $\Omega_{p 2}$ given by equation (8).

We can see that a random (or quasi-random) distribution of dipoles gives the same results as a charge distribution (for $d \ll D$ ). The electric polarizability is given by:

$$
\alpha(\omega)=-\frac{\Omega_{p}^{2}}{4 \pi} \times \frac{1}{\omega^{2}-\Omega_{c}^{2}},
$$

where $\Omega_{p} \gg \Omega_{c}$, and it is identical to the electric susceptibility (since the internal field is very low at the position of the dipoles).

\section{Concluding remarks}

A model of heterogeneous composite material is introduced, and the dynamics governing its electric polarization is discussed. The model consists of randomly distributed micro-domains, either densely packed or highly dispersed, endowed with electric charges or dipoles. The electric polarization is computed for this model and related to the charge displacement or dipole rotation. It is shown that the polarization (and the electric susceptibility) may exhibit characteristic (resonance) frequencies in the radio-frequency range and the material may undergo a displacive modification (translations or rotations) under the action of an external, uniform electric field, very much alike an induced ferroelectric transition. The origin of this change, which may appear even for moderate intensities of the external field and be driven by non-linear microdomain interaction, is the low values of the characteristic radio frequencies. The transition shifts the characteristic frequencies, exhibits a hysteresis loop and is associated with a high dielectric loss. It is very likely that in the static limit the phenomenon investigated here is known as the Maxwell-Wagner-Sillars polarization.

The authors are indebted to the members of the Seminar of the Institute of Atomic Physics and the Laboratory of Theoretical Physics, Magurele, Bucharest, for useful discussions and a careful reading of the manuscript. This work was supported by the Romanian Government Research Agency Grant \#306/SMIS 26614.

\section{References}

1. P.G. de Gennes, Rev. Mod. Phys. 71, S374 (1999)

2. P.M. Chaikin, T.C. Lubensky, Principles of Condensed Matter Physics (Cambridge University Press, New York, 1995), p. 620

3. J.C. Maxwell, Lehrbuch der Elektrizitat und der Magnetismus, vol. 1, Art. 328 (Berlin, 1983)

4. K.W. Wagner, Electr. Eng. 2, 371 (1914)

5. R.W. Sillars, J. Inst. Electr. Eng. (London) 80, 378 (1937)

6. C.J.F. Bottcher, Theory of Electric Polarization (Elsevier, Amsterdam, 1952)

7. F. Kremer, A. Schonhals, W. Luck, Broadband Dielectric Spectroscopy (Springer, Berlin, 2002)

8. Ch. Kittel, Introduction to Solid State Physics, 8th edn. (Wiley, New Jersey, 2005), p. 19

9. D.E. Aspnes, Am. J. Phys. 50, 704 (1982)

10. C. Brosseau, F. Boulic, P. Queffelec, C. Bourbigot, Y. Le Mest, J. Loaec, A. Beroual, J. Appl. Phys. 81, 882 (1997)

11. W.R. Tinga, W.A.G. Voss, D.F. Blossey, J. Appl. Phys. 44, 3897 (1973) 
12. J.C. Garland, D.B. Tanner (eds.), Electric Transport and Optical Properties of Inhomogeneous Media, in AIP Conf. Proc., vol. 40 (American Institute of Physics, New York, 1978)

13. A. Priou (ed.), in Progress in Electromagnetic Research: Dielectric Properties of Heterogeneous Materials, vol. 6 (Elsevier, New York, 1992)

14. W.T. Doyle, J. Appl. Phys. 78, 6165 (1995)

15. B. Sareni, L. Krahenbuhl, A. Beroual, C. Brosseau, J. Appl. Phys. 80, 1688 (1996)

16. B. Sareni, L. Krahenbuhl, A. Beroual, C. Brosseau, J. Appl. Phys. 80, 4560 (1996)

17. Y.I. Dzhezherya, I.Y. Novak, S.P. Kruchinin, Supercond. Sci. Technol. 23, 105011 (2010)

18. S.P. Kruchinin, V.F. Klepikov, V.E. Novikov, D.S. Kruchinin, Mater. Sci.-Poland 23, 1009 (2005)
19. V. Klepikov, S. Kruchinin, V. Novikov, A. Sotnikov, Rev. Adv. Mat. Sci. 12, 127 (2006)

20. L.D. Landau, Phys. Z. Sowjet. 11, 26 (1937)

21. L.D. Landau, Phys. Z. Sowjet. 11, 545 (1937)

22. L.D. Landau, E.M. Lifschitz, Statistical Physics, vol. 5 (Pergamon Press, Oxford, 1980)

23. A.F. Devonshire, J. Phil. Mag. 40, 1040 (1949)

24. A.F. Devonshire, J. Phil. Mag. 42, 1065 (1951)

25. A.F. Devonshire, Adv. Phys. 3, 85 (1954)

26. P.L. Knigt, P.W. Milloni, Phys. Rep. 66, 21 (1980)

Open Access This article is distributed under the terms of the Creative Commons Attribution Noncommercial License which permits any noncommercial use, distribution, and reproduction in any medium, provided the original author(s) and source are credited. 